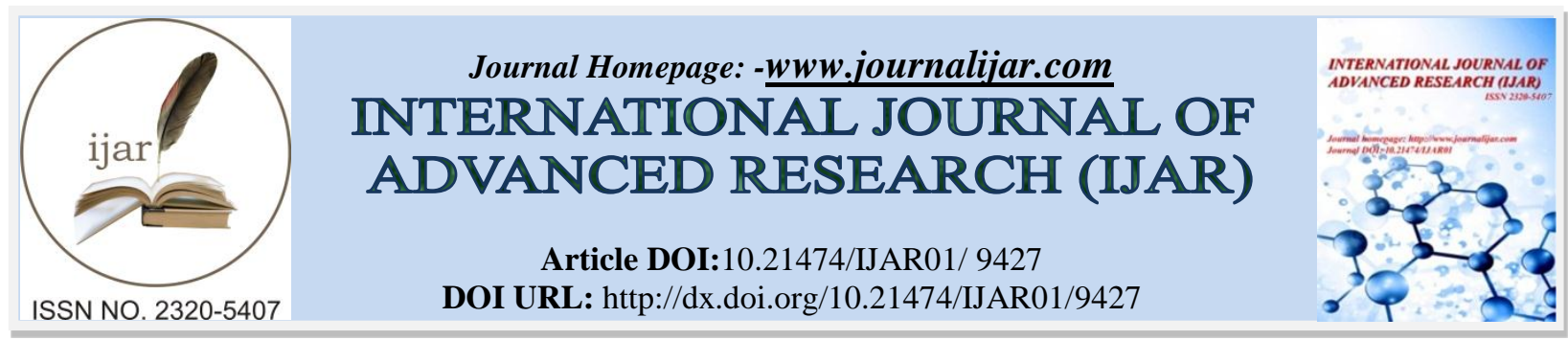

RESEARCH ARTICLE

\title{
DESIGN AND IMPLEMENTATION OF PLC NETWORK SYSTEM USING OPNET MODULATOR.
}

\author{
Raghad s. Majeed ${ }^{1}$, Sahar h.ahmed ${ }^{2}$ and Ahmed h. Ahmed ${ }^{3}$. \\ 1. Medical Instruments Techniques Engineering, Al-Farahidi University, Iraq/Baghdad). \\ 2. Department of Metal Engineering, University of Technology, Iraq/Baghdad. \\ 3. Medical Instruments Techniques Engineering, Al-Farahidi University, Iraq/Baghdad).
}

\section{Manuscript Info}

Manuscript History

Received: 24 May 2019

Final Accepted: 26 June 2019

Published: July 2019

Key words:-

EXM-WIFI PLC, OPNET, Wireless

LAN, Ethernet.

\section{Abstract}

This proposed design explains the methodology of PLC network control system for Industrial application. The programmable logic controller (PLC) is widely used for controlling and monitoring machinery on factory assembly lines, such as controlling upon the process of factory, temperature monitoring, remote maintenance, and factory automation, etc. The PLC network in this paper implemented using OPNET Modeler 14.5 for two schemas Ethernet and Wireless, using EXM-WIFI PLC to evaluate the performance of network such as delay, throughput, network load, and File download response time. OPNET Modeler 14.5 give good assumption on behavior of Ethernet and Wireless network.

Copy Right, IJAR, 2019,. All rights reserved.

\section{Introduction:-}

A PLC network is the generic term that include the hardware, software and procedures used to control and monitor industrial process [1]. This network can perform a real-time, high speed communication with the PLC using a local LAN or Ethernet connection. Fig 1 show the proposed PLC network where the PLC is connected directly to the network using either Ethernet or wireless module. This network module has the great benefit in most industrial application that need real-time monitoring and remote control from long distances: e.g. remote maintenance, building automation, and temperature and voltage monitoring, etc. [2]. The remote control and monitoring of networks have been rising due to the importance of this industrial application in the world. The monitoring and control of a network allows data acquisition in real time. Monitoring provides this information playing an important role in network management [3].

A programmable logic controller is widely used in the industrial control system. PLC is an example of a "hard" realtime system since output results must be produced in response to input conditions within a limited time, otherwise unintended operation will result. [4] The advantages of using PLC are their high reliability, relatively low costs, compact built and module structure which make the further development of a control system possible [5].

In this paper it is presented two kinds of architecture, which can serve as a plan for many remote applications that need wireless or Ethernet networking and evaluate the performance of this two kinds of network. This network has been simulated using OPNET (Optimized Network Engineering Tool) modeler.

Corresponding Author:-Raghad s. Majeed.

Address:-Medical Instruments Techniques Engineering, Al-Farahidi University, Iraq/Baghdad). 
OPNET Technologies is the leading developer of network simulation software and solution provider for application and network management issues. This simulation consists of sixteen scenarios. It allows us to create proposed networks, execute simulations, and analyze the output data [6].

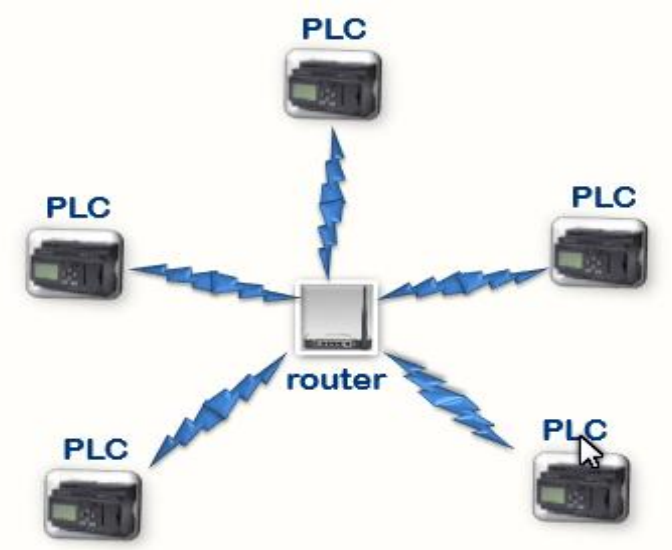

Fig 1:-the proposed PLC network.

\section{Exm-12dc-Da-Rt-Wifi Plc:-}

Easy $\mathrm{x}$-Messenger (EXM) is a compact and expandable unit of measurement combining industrial grade GSM/GPRS modem, PLC controller, data logger, Ethernet module, camera interface, audio output interface and multiple communication capability (USB, RS232 and RS485, MODBUS ASCII/RTU/TCP, Master/Slave). EXM PLC is the good choice for proposed network that required for Ethernet connectivity and wireless communication network. It is supported for $802.11 \mathrm{~b} / \mathrm{g} / \mathrm{n}$ wireless standards [7].

For extra connectivity, these types of Wi-Fi PLC's also incorporate a GSM modem, which provides GPRS/SMS connectivity to your micro PLC control system. Also in smart phone application can be perfectly applied to Wi-Fi PLC [7].

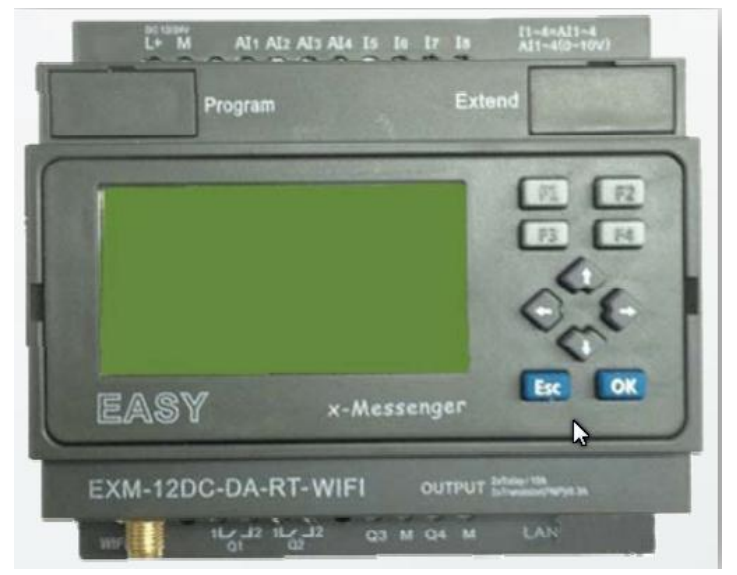

Fig 2:-Easy X-Messenger PLC.

Fig2 show the EXM-12DC-DA-RT-WIFI PLC which has the properties total $12 \mathrm{I} / \mathrm{O}$ pins (4 digital/4 analog input and 2 digital/2 analog), Power Supply (12V DC), Output types (2 Relay(10A) + 2transistors(PNP)/0.3A), and Special function built-in WIFI modem. This properties can be obtained from the naming rules of EXM Series.

The Naming Rules of EXM Series are:

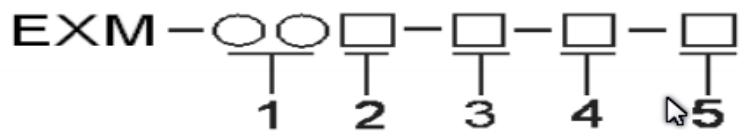




\section{EXM: series name (EASY x-Messenger)}

1. Points of total IOs.

2. Power Supply (DC 12 24V, AC 110 240V).

3. Digital/Analog (D: digital, DA: digital \&analog configurable, DAI: digital, $(0 \ldots 10 \mathrm{~V}) \&(0 / 4 \ldots 20 \mathrm{~mA}))$.

4. Output type (R: Relay TN: transistor (PNP type)).

5. Special function (V: Voice via GSM network/speaker, TV: Voice alarming via wired telephone, C: Webcam connection N: Ethernet access) [7].

The proposed network is mainly used for direct Ethernet and wireless communication (based on the Modbus TCP protocol) between several x-Messenger devices.

\section{Router:-}

The TL-WR741ND 150Mbps Wireless Lite-N Router integrates 4-port Switch, Firewall, NAT-Router and Wireless AP. Powered by $2 \times 2$ MIMO technology, the 150Mbps Wireless Lite-N Router delivers exceptional range and speed, which can fully meet the need of Small Office/Home Office (SOHO) networks and the users demanding higher networking performance.

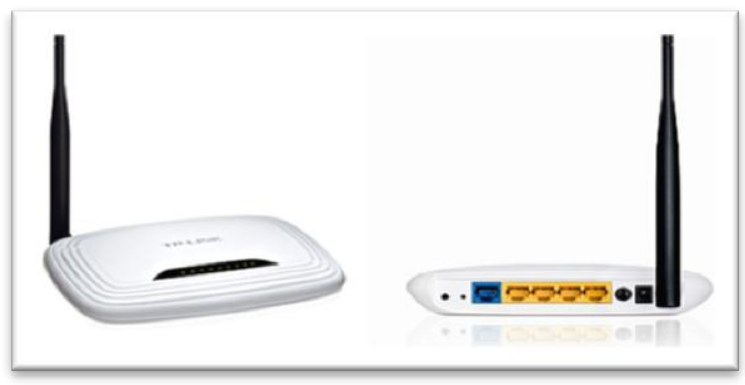

Fig 3:-Tp-link Router

\section{Ethernet (802.3):-}

Ethernet is a very popular LAN technology, relatively inexpensive, and reasonably fast. It has been designed as a broadcast system, i.e., stations on the network can send messages whenever and wherever it wants. All stations may receive the messages, however only the specific station to which the message is directed will respond. It has gone through four generations: Standard Ethernet (10Mbps), Fast Ethernet (100 Mbps), Gigabit Ethernet (1 Gaps), and Ten-Gigabit Ethernet (10 Gaps) [8].

The proposed network system has five PLC and all these PLC's communicate with each other over the Ethernet (802.3). Ethernet 100BaseT is used in proposed network.

\section{Wireless LAN (802.11b):-}

IEEE $802.11 \mathrm{~b}$ describes the high-rate direct sequence spread spectrum (HRDSSS) method for signal generation in the $2.4 \mathrm{GHz}$, HR-DSSS defines four data rates: 1, 2, 5.5, and 11Mbps. The first two use the same modulation techniques as DSSS. The5.5-Mbps version uses BPSK. The 11-Mbps version uses QPSK. "WIFI-EXM PLC" Support $802.11 \mathrm{~b} / \mathrm{g} / \mathrm{n}$ wireless standards and it will operate on $802.11 \mathrm{~b}$ standard with data rate $11-\mathrm{Mbps}$ in proposed network [9].

\section{Performance Analysis Network Layout:-}

In order to study the performance of PLC Ethernet and wireless networks, the network setup have been simulated using OPNET-14.5 simulator. 


\section{Ethernet PLC networks:-}

Fig 2 shows the proposed PLC Ethernet network which consists of one router and five "WIFI-EXM PLC" connected together using Ethernet 100BaseT, the distance between router and each node is 50 meters.

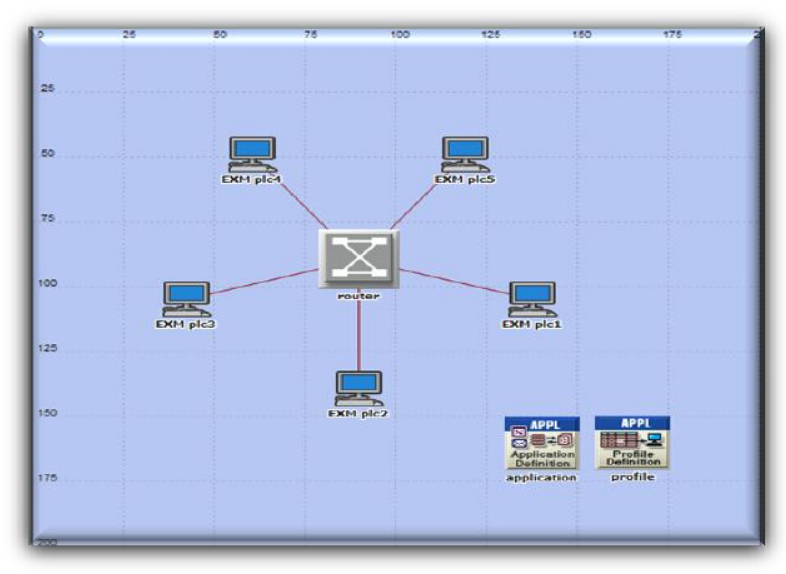

Fig 4:-Ethernet PLC Network

\section{Wireless PLC networks:-}

Fig 1 illustrate the proposed PLC wireless network which consists of one router and five "WIFI-EXM PLC" are connected together using WIFI $802.11 \mathrm{~b}$ with data rate $11 \mathrm{Mbps}$. the distance between router and each node is 50 meters.

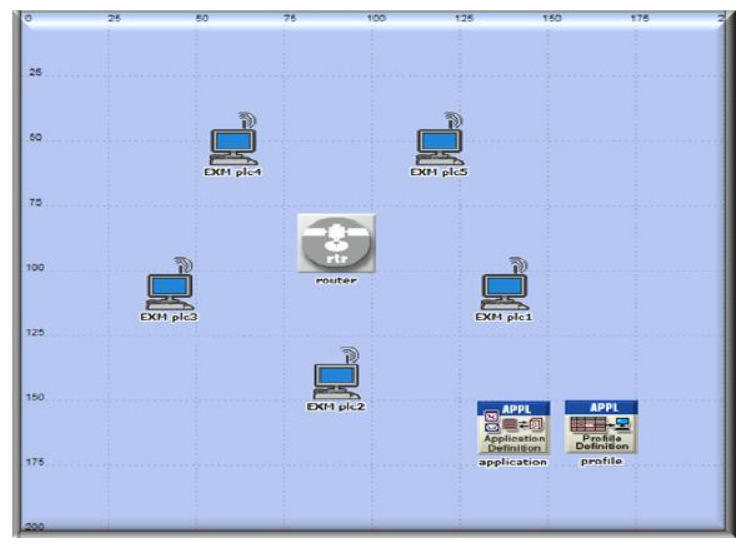

Fig 5:-Wireless PLC Network

Table1 comprise the parameters of EXM PLC1 which define the base station subsystem (BSS) Identifier for communication with router and define the Physical Characteristics of that PLC node which operate with IEEE 802.11b DSSS and Data Rate 11Mbps.

Tabel 1:-Exm Plc1 Parameters

\begin{tabular}{|l|l|}
\hline Attribute & Value \\
\hline BSS Identifier & 192.168 .1 .11 \\
\hline Access Point Functionality & disable \\
\hline Physical Characteristics & Direct Sequence \\
\hline Data Rate & $11 \mathrm{Mbps}$ \\
\hline
\end{tabular}

\section{Performance Results:-}

The performance results of Ethernet and wireless PLC networks represent by delay, throughput, network load, File download response time.Table2 and Table3 comprise the parameters of Application and Profile configuration of two scenarios. 
Tabel 2:-Application configuration parameters

\begin{tabular}{|l|l|}
\hline Attribute & Value \\
\hline Application configuration & $(\ldots)$ \\
\hline Number of row & 1 \\
\hline Application name & PLC file transfer (ftp) \\
\hline File size (byte) & constant (135000) \\
\hline
\end{tabular}

Tabel 3:-Profile configuration parameters

\begin{tabular}{|l|l|}
\hline Attribute & Value \\
\hline Profile configuration & $(\ldots)$ \\
\hline Number of row & 1 \\
\hline Profile name & PLC ftp prof \\
\hline Operation mode & Serial (random) \\
\hline Start Time (seconds) & Uniform (100,110) \\
\hline stop Time (seconds) & End of simulation \\
\hline
\end{tabular}

\section{Delay:-}

The latency or delay defines how long it takes for an entire message to completely arrive at the destination from the time the first bit is sent out from the source. Fig 6 shows the comparison between the delay at EXM PLC1 node in two proposed networks (PLC Ethernet and wireless network) which. As expected, delay in EXM PLC1 node for wireless network greater than the delay for same node in Ethernet network. The delay for Ethernet network is begins from 0.3 second, after that it keeps on increasing to reach 0.0058 second, while the delay for wireless network is ranges from 0 second to 0.0004 second.

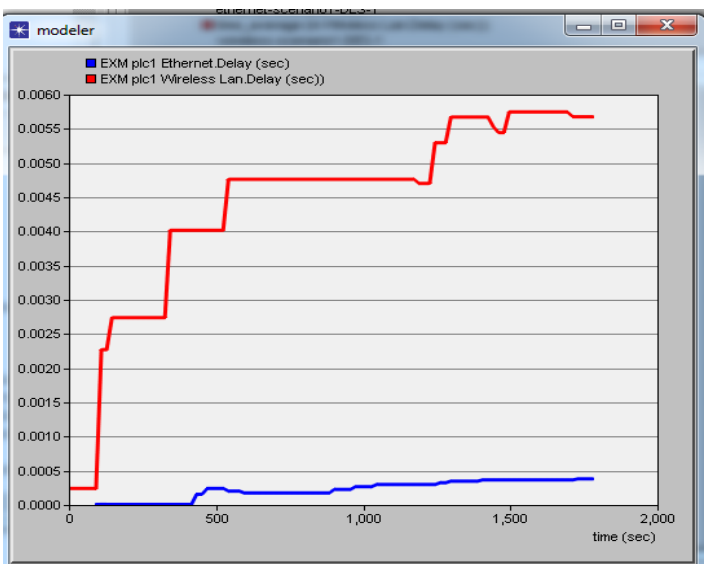

Fig 6:-Delay at EXM PLC1

The comparison of global statistics for PLC Ethernet and wireless network delay can be shown in Fig 7.

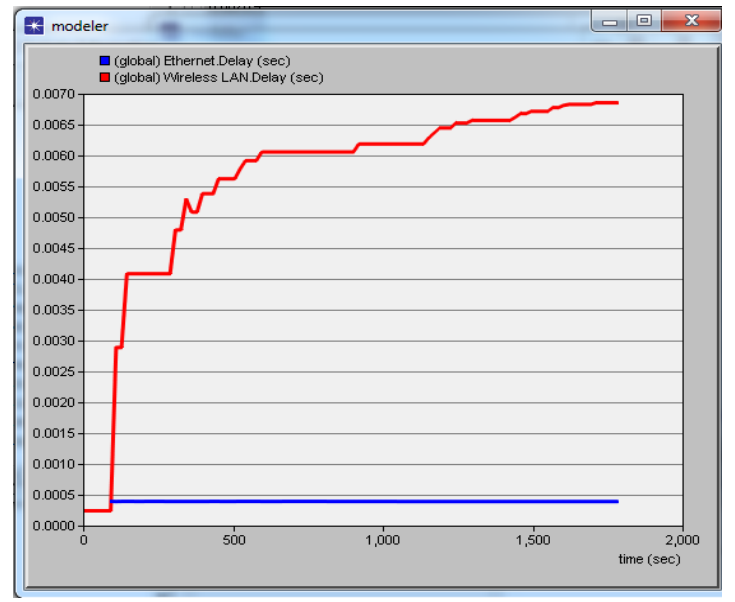

Fig 7:-Delay on global network 


\section{Throughput:-}

Throughput is the average rate of successful message delivery over a communication channel. The throughput is usually measured in bits per second (bps) or in data packets per second. Fig 5 shows the comparison between the Throughputs at EXM PLC1 node in tow proposed networks.

From Fig 5 it is observed that the Throughput of Ethernet network greater than the Throughput of wireless network. Ethernet throughput begins from the value, after some time it decreases then it keeps on increasing and decreasing within specific range, wireless throughput is smaller than Ethernet throughput. Where the $\mathrm{x}$-axis represent time in second and y-axis represent data rate in (bps).

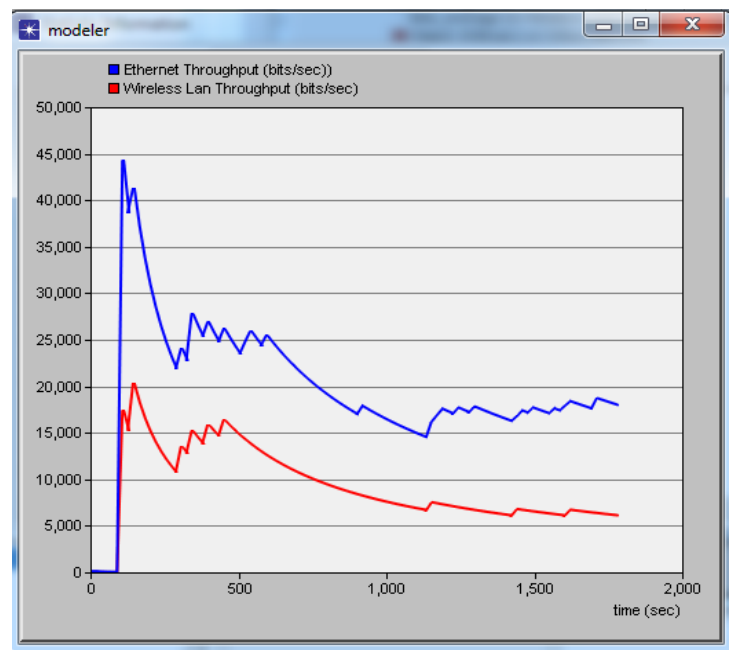

Fig 8:-Throughput

\section{Network Load:-}

Fig 6 illustrate the comparison between the loads on two networks, from this graph notice that the load on Ethernet network is greater than on wireless network. Where the $\mathrm{x}$-axis represent time in second and $\mathrm{y}$-axis represent data rate in (bps).

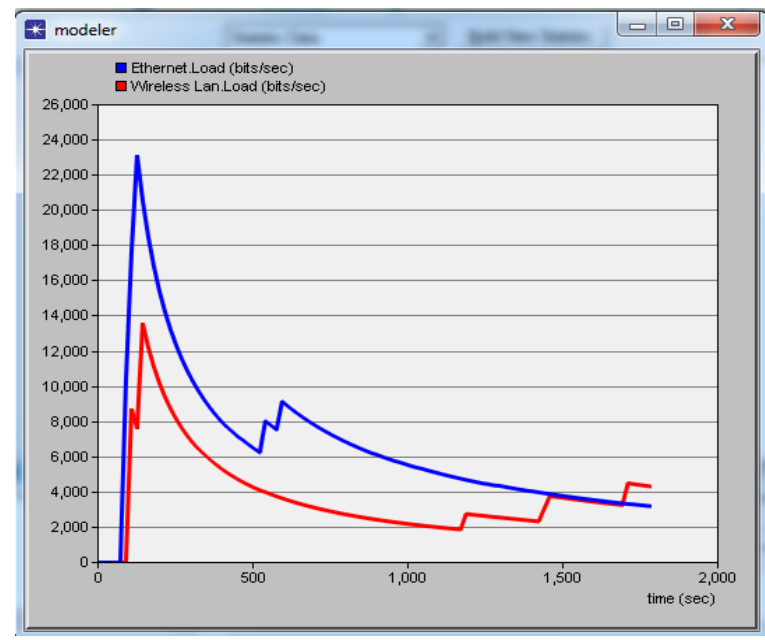

Fig 9:-network load 
File download response time:-

Finally, Fig 7 shows the time for download file in EXM PLC1 node in two scenarios. File need about 0.50 second to download in EXM PLC1 node in wireless network which is greater than the time need for download on same node for Ethernet network which is about 0.15 second.

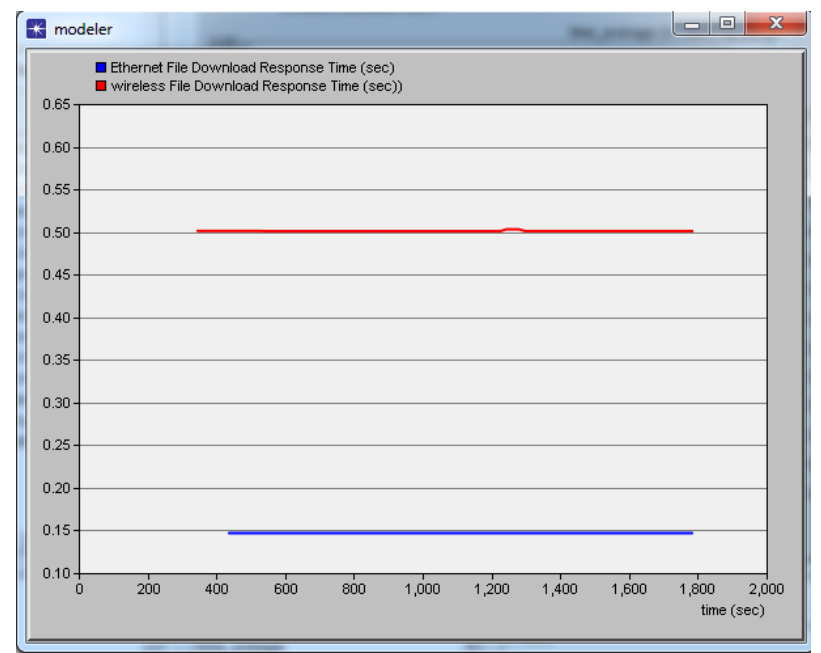

Fig10:-File Download response time at EXM PLC1

\section{Conclusion:-}

From the results of proposed system for Ethernet and wireless PLC networks, the performance is evaluated using OPNET Modeler. By analyzing all the graphs, the results of Ethernet scheme observed that the delay, throughput, network load, and File download response time of WIFI-EXM PLC are very good behavior but the limitation of Ethernet scheme is the distance dependence which is Proportional to an inverse relationship. On the other hand the results of wireless scheme observed that the delay, throughput, network load, and File download response time of WIFI-EXM PLC are less behavior but the distance not represent the limitation of this scheme.

\section{References:-}

1. Anjali S. Ashtekar, Bhagsen J.Parvat and Chandrakant B. Kadu, "Application of MODBUS to Communicate the PLC," International Journal of Emerging Science and Engineering (IJESE), vol. 1, no. 11, pp. 41-45, 2013.

2. N. Gal, "Remote Ethernet PLC Networking and Control," in 17th Telecommunications forum TELFOR, Serbia, Belgrade, 2009.

3. C. Leão, F. Soares, J. Machado, E. Seabra and H. Rodrigues, "Design and Development of an Industrial," International Journal of Engineering and Technology (IJET), vol. 6, no. 1, pp. 21-26, 2011.

4. "Wikipedia," [Online]. Available: http://en.wikipedia.org/wiki/Programmable_logic_controller. [Accessed 1 may 2014].

5. Ł. Ścisło, "CONTROLLER-SENSING ELEMENT COMMUNICATION USING UDP PROTOCOL".

6. Adarshpal S. Sethi and Vasil Y. Hnatyshin, the Practical OPNET® User Guide for Computer Network Simulation, New York: Taylor \& Francis Group, 2013.

7. [Online]. Available: www.xlogic-relay.com/x-Messenger\%20manual.pdf. [Accessed 2 may 2014].

8. E. P. K. Er. Ishu Gupta, "Comparative Throughput of WiFi \& Ethernet LANs using," International Journal of Computer Applications, vol. 8, no. 6, pp. 6-9, 2010.

9. Behrouz A.Forouzan and Sophia Chung Fegan, Data Communicationsand Networking, New York: McGrawHill Forouzan networking, 2007. 